\title{
Influence of Laser Beam Shaping on Melt Pool Thermocapillary Flow
}

\author{
Seyyed Mohammad Ali Noori Rahim Abadi, Yongcui Mi, Fredrik Sikström, Isabelle Choquet \\ University West \\ Department of Engineering Science, 46186 Trollhättan, Sweden \\ ali.abadi@hv.se, yongcui.mi@hv.se, fredrik.sikstrom@hv.se, isabelle.choquet@hv.se
}

\begin{abstract}
The effect of different shapes of laser beam power density distribution was investigated numerically with respect to the thermo-hydrodynamics of the melt pool during welding. The process addressed is conduction mode bead on plate welding of the Titanium alloy Ti-6Al-4V. A new solver based on the volume of fluid method to track the deformation of the melt free surface was developed in the OpenFOAM software. Experiments were conducted for the purpose of validating the model. In addition to the traditional cross-cut images of the weld bead, top view images of the melt pool were analysed to perform the validation along the 3space dimensions. A good agreement between numerical predictions and experimental measurements was obtained, thus promising a confident utilization of this simulation model when investigating the influence of beam shapes on the resulting weld seam. The effect of three different beam shapes on the melt pool velocity flow, temperature fields, and melt geometry were studied. It was found that the melt pool size was largest for an elliptical power density distribution with the major axis along the welding direction. The results also showed that the laser beam with Gaussian power density distribution resulted in the deepest penetration.
\end{abstract}

Keywords: Thermocapillary Flow, Free surface deformation, Laser beam shaping, Welding, VOF, OpenFOAM, Validation.

\section{Introduction}

Laser beam processing is a common method in e.g. joining and additive manufacturing of materials like metals and is very popular in many industries such as automotive and aerospace. It can provide a precise weld seam with a minimal heat affected zone (HAZ). Since the laser beam spot size can be tuned it can create deep and narrow welds with optimum heat input, which results in parts with low residual heat deformation. Although Laser beam welding (LBW) has many advantages it can also present some detrimental defects such as cracking, undercut or too narrow weld and could thus benefit from beam shaping [1-3]. Beam shaping can indeed be used to tune the process and potentially improve properties of the resulting weld seam [4]. Thermo-hydrodynamics could be used to predict the effect of beam shaping on the melt pool and provide process knowledge not yet accessible to measurements.

Laser beam welding is governed by a complex transient and multiphase physics that encompass melting, solidification, evaporation and possible material vaporization, heat conduction, convection and radiation, and thermocapillary flow [5]. Many studies have been conducted to expand the knowledge about LBW considering the aforementioned phenomena both experimentally $[6,7]$ and numerically $[8,9]$. There co-exist different levels of modelling at different levels of accuracy, regarding the numerical simulation. For instance, Akbari et al. [10] studied LBW of Ti-6Al-4V both numerically and experimentally ignoring the deformation of the free surface in their numerical simulations. They performed a validation based on comparison between simulation and experimental cross-sections, and temperature measurements with k-type thermocouples. A maximum deviation of $17 \%$ between the numerical results and experimental data was reported in their works. They also found, as expected, that the penetration depth and weld width increased with decrease in the welding travel speed. Although the changes in material properties with respect to temperature can considerably affect the temperature and flow fields during LBW, in some studies, it has been neglected [7, 11]. Kidess et al. [12] studied the flow instabilities during laser welding of steel ignoring the variations of material properties, except the surface tension, with respect to temperature. They examined the performance of both Direct numerical Simulation (DNS) and Large Eddy Simulation (LES). Moreover, different flow regimes were distinguished based on the thermal Marangoni force. In another work, Kidess et al. [13] investigated the development of shape and grain morphology during LBW with combining the macroscale surface tension driven fluid flow and heat transfer model and a mesoscale model based on solidification kinetics. They used a fixed positioned laser for the simulations; such welding process is used in spot welding application. 
Their results showed that the Marangoni force was governing the weld pool shape and grain morphology. There are also some advanced research works in which many detailed physics are taken into account $[14,15]$.

To our knowledge the effect of different shapes of the laser power density distribution on the melt pool thermohydrodynamics has not been studied before in numerical simulation. To investigate this problem, a solver based on the VOF method [16] is further developed in the OpenFOAM software. The solver is capable of simulating the transient and multiphase physics of welding process assuming negligible vaporization and including the following aspects: deformation of the melt pool free surface, variable material properties with respect to temperature, convection effect in the melting and solidification process, and laser motion. Validation experiments have been conducted to assess the performance and accuracy of the developed solver. At this stage, the validation experiments are limited to the beam shape using a top hat beam. Three different welding travel speed setups are evaluated from perpendicular cross-cut images of the resulting weld seam, supplemented with top surface images of the melt pool during welding of the Titanium alloy, Ti-6Al-4V. Three relevant cases of beam shapes are then evaluated in conduction mode bead on plate welding of this Titanium alloy; case 1 uses an elliptical distribution with the major axis perpendicular to the welding direction, case 2: uses an elliptical distribution with major axis along the welding direction, and case 3: uses a symmetrical Gaussian distribution.

\section{Mathematical model}

A transient and three-dimensional one-fluid model (also called mixture model) assuming laminar flow for Newtonian and incompressible fluid is applied. It is supplemented with an algebraic volume of fluid (VOF) method to predict the deformation of the gas-metal interface [15]. It is also combined with a mushy zone model for the solidliquid metal transition. The equations governing the one-fluid mass, momentum and thermal energy per unit volume and the metal volume fraction $\alpha$ are expressed as [9]

$$
\begin{gathered}
\partial_{t} \rho+\nabla \cdot(\rho \vec{U})=0 \\
\partial_{t}(\rho \vec{U})+\nabla \cdot(\rho \vec{U} \vec{U})=-\nabla p+\nabla \cdot(\mu \nabla \vec{U})+\vec{S}_{U} \\
\partial_{t}\left(\rho C_{p} T\right)+\nabla \cdot\left(\rho C_{p} T \vec{U}\right)=\nabla \cdot(\kappa \nabla \mathrm{T})+S_{T}+Q_{l} \\
\partial_{t} \alpha+\nabla \cdot(\alpha \vec{U})+C_{\alpha} \nabla \cdot\left(\alpha(1-\alpha) \vec{U}_{r}\right)=0
\end{gathered}
$$

This set of equations is supplemented with the source terms:

$$
\begin{gathered}
\vec{S}_{U}=\left[\sigma k \vec{n}+\frac{d \sigma}{d T}(\nabla T-\vec{n}(\vec{n} . \nabla T))\right]|\nabla \alpha| \frac{2 \rho}{\rho_{l}+\rho_{g}}+\rho \vec{g}\left[1-\beta\left(1-T_{m}\right)\right]-C_{D} \frac{\left(1-f_{l}\right)^{2}}{f_{l}^{3}+\varepsilon_{D}} \vec{U} \\
S_{T}=\Delta h_{f}\left[\partial_{t}\left(\rho f_{l}\right)+\nabla \cdot\left(\rho \vec{U} f_{l}\right)\right] \\
Q_{l}=\frac{2 \eta P_{l}}{\pi a b} \exp \left[-2\left(\frac{x-U_{\text {laser }} t}{a}\right)^{2}-2\left(\frac{y}{b}\right)^{2}\right] \nabla \alpha
\end{gathered}
$$

In these equations the one-fluid density, $\rho$, the viscosity, $\mu$, the specific heat capacity, $C_{p}$, and the thermal conductivity, $\kappa$, are expressed as functions of the respective properties of the gas $(g)$, and the metal in liquid $(l)$ and solid $(s)$ state, as well as the metal volume fraction and the liquid fraction of metal, $f_{l}$ [9]. This last one depends on temperature and is expressed according to Rössler and Brüggeman [17]. Furthermore, $t$ denotes the time, $\vec{U}$ the one-fluid velocity, $p$ the pressure, and $T$ the temperature. $C_{\alpha}$ and $\vec{U}_{r}$ are the compression factor and compression velocity, respectively; they ensure the sharpness of the interface [18]. The momentum source term, $\vec{S}_{U}$, includes (from left to right) surface tension, body forces and Darcy damping force [9]. $\sigma$ is the surface tension, $k$ is the interface curvature, $\vec{n}$ is the normal to the interface, $\vec{g}$ is the gravitational acceleration, $\beta$ the volumetric expansion coefficient; $C_{D}$ is a large constant $\left(=10^{8}\right)$, and $\varepsilon_{D}$ is a small constant $\left(=10^{-3}\right)$ for avoiding the division by zero. The index $m$ holds for melting condition set as the average between solidus and liquidus states. The power source term, $S_{T}$, is calculated as in [17], and $\Delta h_{f}$ is the latent heat of fusion. The laser power 
density distribution, $Q_{l}$, is a function of the laser absorption coefficient, $U_{\text {laser }}$ is the laser speed, $\eta=0.34$ [19], and the laser power, $P_{l}=1 \mathrm{~kW} ; x$ and $y$ are coordinate directions. The geometrical parameters $a$ and $b$ are used to set the laser power density distribution; case 1: $a=0.65 \times 10^{-3} \mathrm{~m}, b=2.5 \times 10^{-3} \mathrm{~m}$ (Elliptical, major axis perpendicular to the welding direction), case $2: a=2.5 \times 10^{-3} \mathrm{~m}, b=0.65 \times 10^{-3} \mathrm{~m}$ (Elliptical, major axis along the welding direction), and case 3: $a=$ $0.65 \times 10^{-3} \mathrm{~m}, b=0.65 \times 10^{-3} \mathrm{~m}$ (Gaussian).

Figure 1 shows the computational domain of size $40 \times 12 \times 4 \mathrm{~mm}$ in $x, y$, and $z$ directions, respectively. This domain contains a $2 \mathrm{~mm}$ thick flat plate made of Ti-6Al-4V that is covered with argon gas. The Ti-alloy has temperature dependent properties that can be found in refs. [20,21], while the rare gas has constant properties. Due to the symmetry only half of the real plate is simulated. Except the symmetry plane, all the boundary faces related to the gas phase are set as pressure inlet/outlet boundary conditions, and at the ambient temperature of $293 \mathrm{~K}$. At the boundary surfaces of the plate, which are cooled by natural convection $\left(h_{c}=20 \mathrm{~W} /\left(\mathrm{m}^{2} . \mathrm{K}\right)\right)$ and radiation with temperature dependent emissivity coefficient, the velocity is set to zero. The domain is discretized into non-uniform hexahedral cells. After performing a grid study, a grid with 512000 cells is used for the simulations. The grid is very fine at the vicinity of the melt pool (10 cells per millimeter).

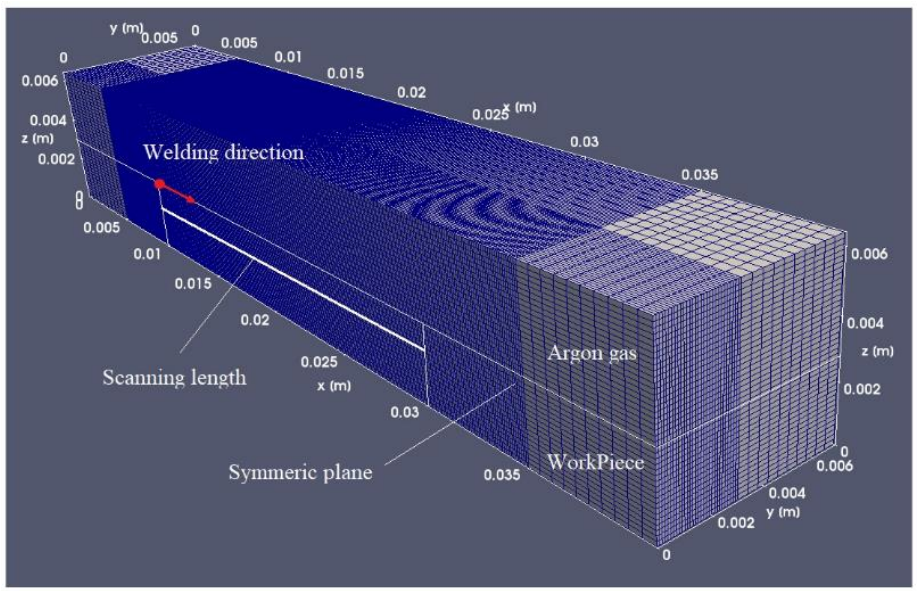

Fig. 1: Computational domain.

The set of governing equations was implemented in the OpenFOAM software based on the original built-in solver interfoam [16, 18]. The Pressure-Implicit with Splitting of Operators (PISO) algorithm is used to handle the pressurevelocity coupling. The Multidimensional Universal Limiter with Explicit Solution (MULES) technique [18] is utilized to solve the volume fraction equation. In order to obtain a stable solution, the CFL number is set to 0.2, which resulted in variations of time step ranging from $10^{-6}$ to $10^{-4} \mathrm{~s}$. Furthermore, the numerical computations continued until 2 seconds in which the flow and temperature fields reach to stable conditions. This was also confirmed in the experimental observations.

\section{Validation Experiments}

To evaluate the predictive performance of the model, bead on plate with three welding travel speeds of $U_{l}=5 \mathrm{~mm} / \mathrm{s}$, $7.5 \mathrm{~mm} / \mathrm{s}$ and $10 \mathrm{~mm} / \mathrm{s}$ were conducted and monitored by a vision camera. As shown in Figure 2, the LBW equipment consists of a water-cooled high-power ytterbium-doped fibre laser of wavelength $1070 \mathrm{~nm}$, a CNC gantry, a welding chamber, fixtures, and a camera vision system. The chamber provides an inert argon environment with oxygen level below $0.2 \%$. The laser beam spot size, defined for Gaussian beam as the radial distance from the centre point of maximum irradiance to the $1 / \mathrm{e}^{2}$ point, is $1.5 \mathrm{~mm}$ on the focal plane. The beam with $1 \mathrm{~kW}$ continuous wave power was focused on the workpiece to conduct the welding. A camera vision system was integrated into the processing optics for top view monitoring coaxial to the laser beam and calibrated to measure the melt pool geometries, see Figure 2 (b). The welding processes were recorded with a frame rate of 200 frames/s. Each weld has a length of $50 \mathrm{~mm}$. 
Figure 3 shows top view images of the melt pool for the three welding setups. A clearly visible boundary can be seen between the liquid metal and the solidifying bead. Surrounding the bright laser spot, some other bright spots can be observed in the melt pool and the solidified bead. Due to the uneven illumination (see LEDs in Figure 2), the front part of the melt pool is sometimes not clearly captured, but its trailing part clearly shows the tail of the melt pool. The width and length of the melt pool (in the plane $z=0$ ) are measured by the vision system with an estimated uncertainty of $\pm 0.1 \mathrm{~mm}$. Moreover, several cross-cut samples from each of these setup welds were mounted in a thermoplastic resin, ground and polished; see left part of Figure 4. The shape of the fusion zone (FZ) and HAZ were revealed with an electrochemical etching and then digitally captured with a camera in an optical microscope.
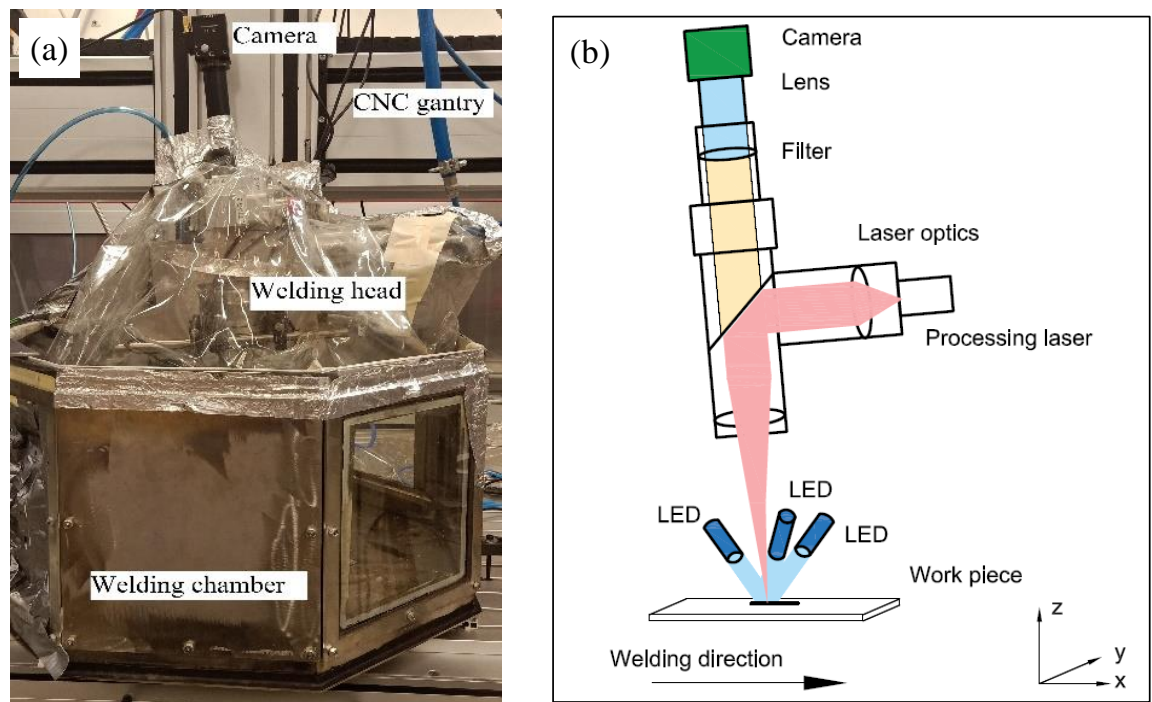

Fig. 2: Welding setup; (a) Welding chamber, (b) Setup components arrangement.
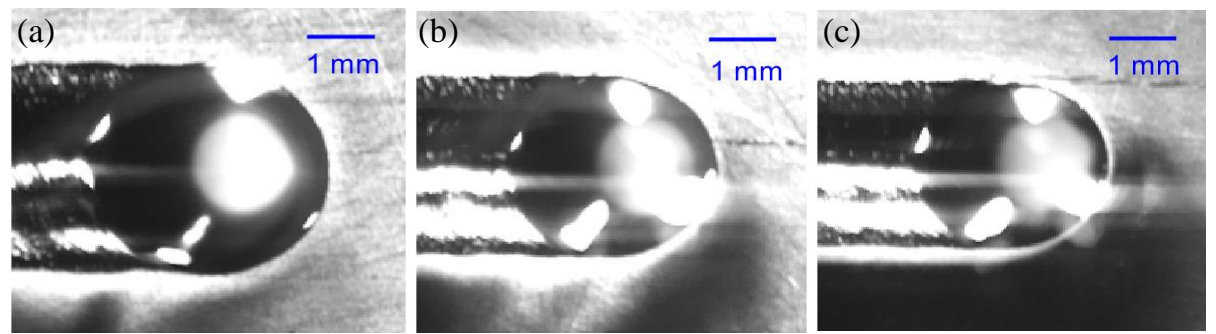

Fig. 3: Top view of melt pool (welding direction is from left to right);

(a) $U_{l}=5 \mathrm{~mm} / \mathrm{s}$, (b) $U_{l}=7.5 \mathrm{~mm} / \mathrm{s}$, (c) $U_{l}=10 \mathrm{~mm} / \mathrm{s}$.

\section{Results and Discussion}

\subsection{Model Validation}

Figure 4 shows the comparison between the numerical results and a corresponding cross-cut micrograph of the weld. It can be observed that the weld geometry is typical of conduction mode welding, as expected at low laser power density $\left(0.56 \mathrm{~kW} / \mathrm{mm}^{2}\right)$ were vaporization of working material is negligible [22]. It can be clearly seen that the developed solver is well-capable of predicting the two different temperature range regions characterizing the FZ and the HAZ. The FZ, corresponding to the melt pool, is recognizable by very large grain sizes while the HAZ coincides with smaller grains. In these regions the material properties of Ti-6Al-4V change considerably with respect to the temperature. 
Table 1 compares the numerical results and experimental measurements for melt pool sizes for $U_{1}=7.5 \mathrm{~mm} / \mathrm{s}$. The predictions are in an acceptable range compared to the experimental data. The maximum deviation between the numerical results and the experimental measurements is $12.5 \%$.

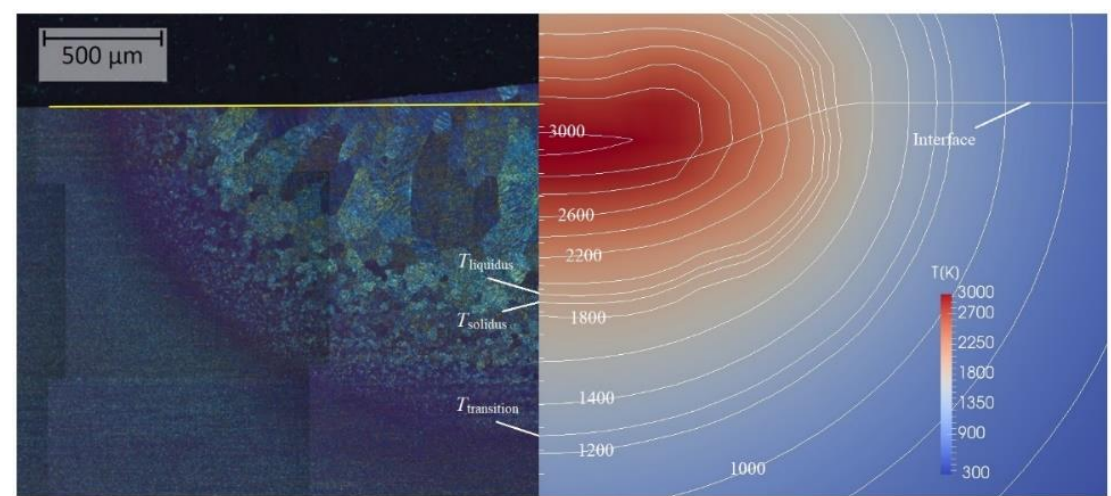

Fig. 4: Comparison between experimental micrograph (left) and numerical simulation results (right) for $U_{1}=7.5 \mathrm{~mm} / \mathrm{s}$ (Isotherm lines vary from $600 \mathrm{~K}$ to $3000 \mathrm{~K}$ with an increment of $200 \mathrm{~K}$ ).

Table 1: Comparison between predicted and experimentally measured melt pool dimensions for $U_{1}=7.5 \mathrm{~mm} / \mathrm{s}$.

\begin{tabular}{|c|c|c|}
\hline & Numerical results (mm) & Experimental data (mm) \\
\hline Melt depth & 0.87 & $0.9 \pm 0.1$ \\
\hline Melt width & 2.45 & $2.8 \pm 0.1$ \\
\hline Melt length & 3.31 & $3.1 \pm 0.1$ \\
\hline
\end{tabular}

Figure 5 shows the evolution of the melt pool and melt velocity along time for case 2 and $U_{1}=7.5 \mathrm{~mm} / \mathrm{s}$. The red color represents the liquid metal fraction and the blue color the solid region. It is observed that the melt pool reaches to a stable condition after approximately one second, even though there are some fluctuations in the trailing part of the melted region. These fluctuations are prevalent in the simulation of free surface flow with VOF method which also has its diffusion issue at the interface [23]. Furthermore, a vortex can be distinguished in the trailing part of the melt pool which can be attributed to the motion of laser beam from the left to the right direction and also the thermal Marangoni force. These effects cause a circular flow pattern which start from the location of laser and ends at the behind of melt pool were the melted materials is solidifying.
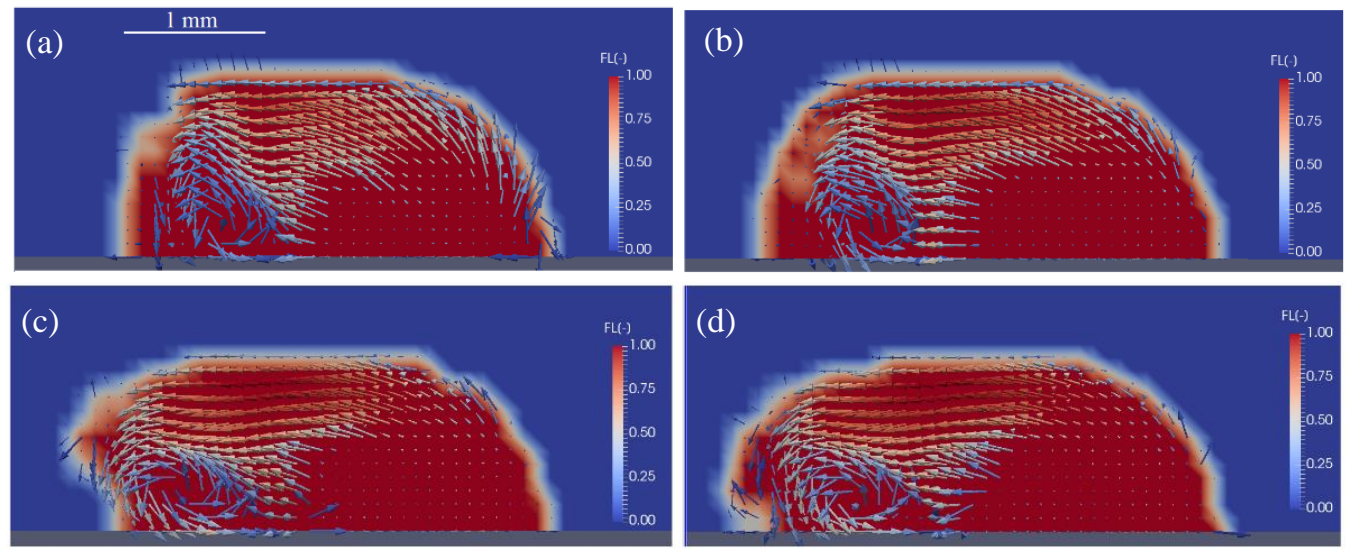

Fig. 5: Evolution of melt pool and velocity field for case 1 and $U_{\mathrm{l}}=7.5 \mathrm{~mm} / \mathrm{s}$; (a) $t=0.5 \mathrm{~s}$, (b) $t=1.0 \mathrm{~s}$, (c) $t=1.5 \mathrm{~s}$, (d) $t=2.0 \mathrm{~s}$. 


\subsection{Influence of Laser Beam Shaping}

Figure 6 illustrates the effect of beam shaping on the melt pool for $U_{1}=7.5 \mathrm{~mm} / \mathrm{s}$ at $t=2.0 \mathrm{~s}$. In Figure 6 (a) the results are plotted in the symmetry plane. It can be seen that the size of the melt pool is the largest for case 2 in which the laser power density distribution is elliptical with major axis along the welding direction. As the laser spot is elongated along the welding direction, its leading part pre-heats the workpiece before the central part of the beam melts the material. Also, its trailing part can provide longer time for heating thus enhance temperature diffusion. Furthermore, Figure 6(a) shows that the penetration depth is the highest for case 3 (Gaussian), since then the laser power intensity is the highest due to the smallest laser spot area. Figure 6(b) depicts the melt pool contours in a cross section normal to the welding direction. The results clearly show that the case 1 has the highest weld width due to the highest laser spot elongation perpendicular to the welding direction. It is also worth mentioning that the deformation of the free surface is the most pronounced for case 3. As the laser spot size is then the smallest, the laser power per unit area is then the highest. This results in forming a region with higher temperature compared to the cases 1 and 2 . The consequent higher temperature gradients cause larger deformation of the free surface due to higher magnitude of the thermocapillary (or Marangoni) force.
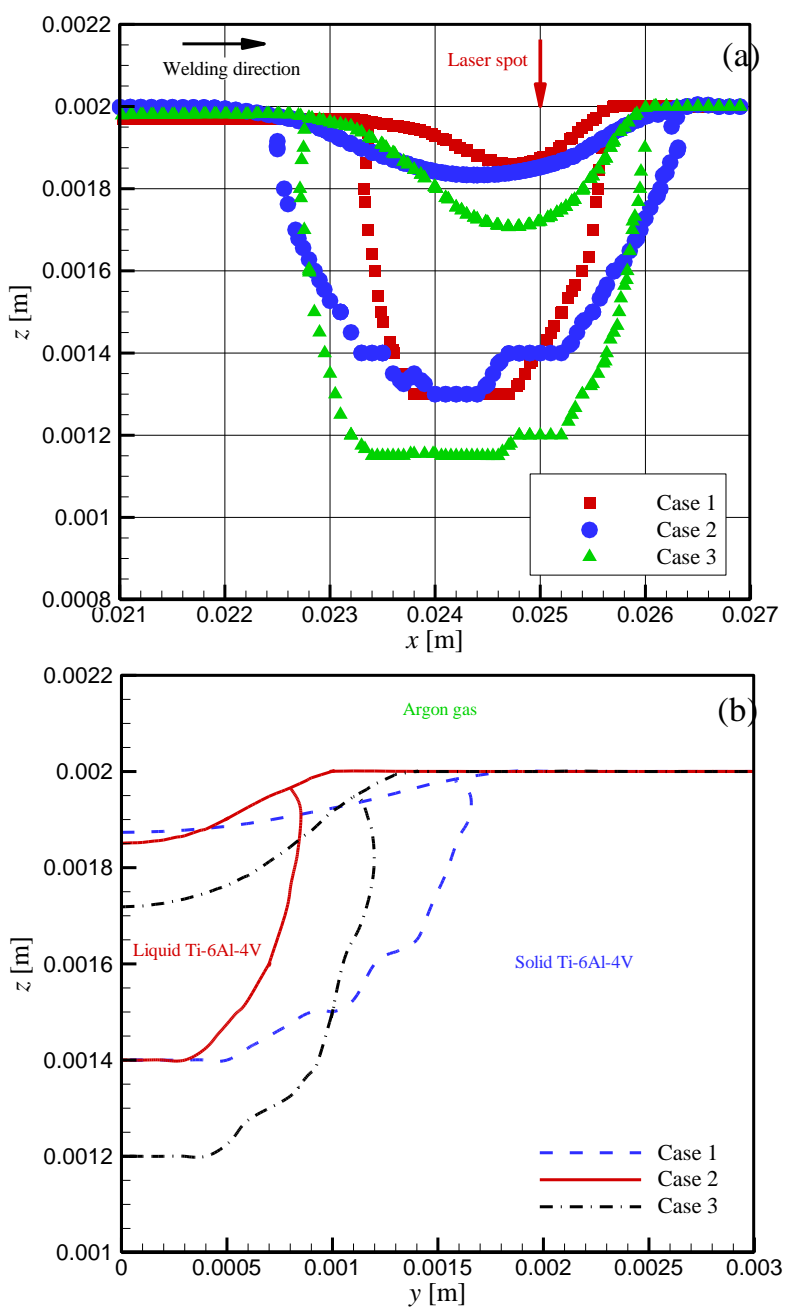

Fig. 6: Effect of laser heat distribution shape on the free surface deformation and melt front line, (a): in the symmetry plane and (b): in a cross-section, at $t=2.0 \mathrm{~s}$ and $U_{1}=7.5 \mathrm{~mm} / \mathrm{s}$. 
In Figure 7 similar results are presented but for different welding travel speeds. These results are plotted at the symmetry plane for case 1 and at $t=2.0 \mathrm{~s}$. They show that the increase in the welding travel speed causes a lowering of the melt pool length and penetration depth. This is expected as the change in heat input per unit welded length is inversely proportional to the change in welding speed as the laser power is constant. Furthermore, higher welding travel speed can also contribute to temperature diffusivity decrease. Besides, Figure 7 shows that the deformation of the free surface increases when the welding travel speed decreases, because it leads to the formation of a hotter free surface. As underlined above, a hotter free surface results in larger temperature gradients and thus higher thermocapillary force.

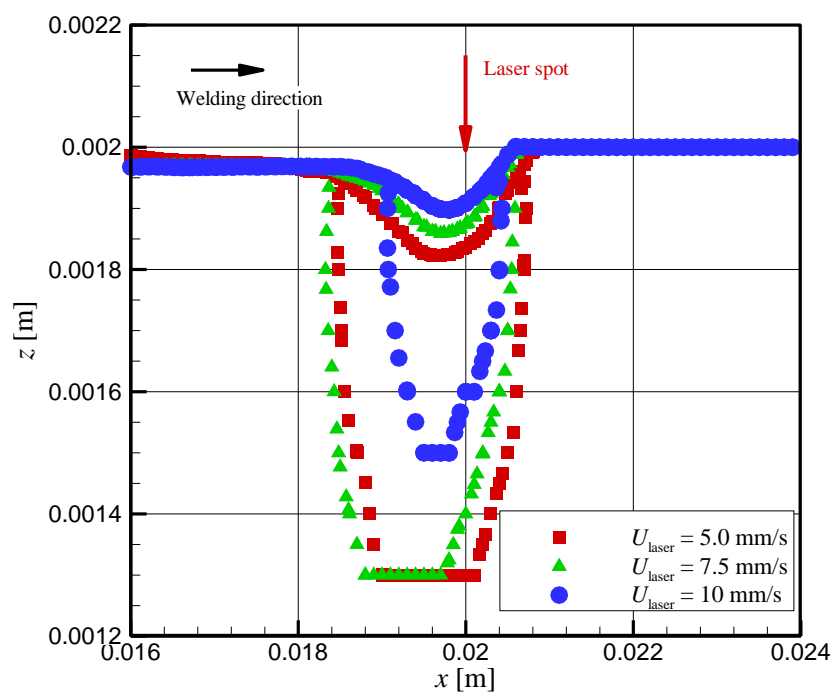

Fig. 7: Effect of welding travel speed on the free surface and melt front line for case 1 at $t=2.0 \mathrm{~s}$.

\section{Conclusions and Future Work}

The first result of this work is a predictive model useful for studying the influence of laser beam power distribution shape on melt pool thermo-hydrodynamics in LBW. A novel way to validate the model was applied, supplementing the traditional experimental cross-cut micrographs by top view images of the melt pool recorded during the experiments and analysed to extend the characterization of the melt pool size to the 3 -space dimensions. The numerical results show a good agreement with the experimental data. The maximum deviation is $12.5 \%$ in the melt pool length at the welding travel speed of $7.5 \mathrm{~mm} / \mathrm{s}$. The results from the three beam shapes evaluated at the same laser power show that the Gaussian laser beam results in the deepest penetration. The elliptical laser beam with the major axis along the welding direction resulted in the largest melt pool length. The predictive performance of the model will enable effective tailoring of the laser beam shape to specific welding and additive manufacturing applications and issues such as gap bridging in butt joint welding, reaching a required seam size and shape, or influencing the microstructure through the temperature gradients and cooling rates.

\section{Acknowledgements}

This research work is supported by grants from the Swedish Knowledge Foundation projects AdOpt (20170315) and SAMw (20170060), which is gratefully acknowledged. The authors would also like to thank Dr. Morgan Nilsen for his technical support during experiments and Karthikeyan Thalavai Pandian for his support in preparing the cross-cut images of the welds. 


\section{References}

[1] B. Victor, D. F. Farson, S. Ream, and C. T. Walters, "Custom beam shaping for high-power fiber laser welding, "Welding J., vol. 90, pp. 113-120, 2011.

[2] K. S. Hansena, M. Kristiansen, and F.O. Olsen, "Beam shaping to control of weld pool size in width and depth," Phys. Proc., vol. 56, pp. 467-476, 2014.

[3] J. Khare, R. Kaul, P. Ganesh, H. Kumar, R. Jagdheesh, and A. K. Nath, "Laser beam shaping for microstructural control during laser surface melting,” J. Laser Appl., vol. 19, pp. 1-7, 2007.

[4] M. Rasch, C. Roider, S. Kohl, J. Strauß, N. Maurer, K. Y. Nagulin, and M. Schmidt, "Shaped laser beam profiles for heat conduction welding of aluminium-copper alloys," Opt. Lasers Eng., vol. 115, pp. 179-189, 2019.

[5] T. Debroy and S. A. David, "Physical Processes in Fusion-Welding," Rev. Mod. Phys., vol. 67, pp. 85-112, 1995.

[6] M. J. Tobar, M. I. Lamas, A. Yáñez, J. M. Sánchez-Amaya, Z. Boukha, and F. J. Botana, "Experimental and simulation studies on laser conduction welding of AA5083 aluminium alloys," Phys. Proc., vol. 5, pp. 299-308, 2010.

[7] W. Pitscheneder, T. DebRoy, K. Mundra, and R. Ebner, "Role of sulfur and processing variables on the temporal evolution of weld pool geometry during multikilowatt laser beam welding of steels," Weld. J., vol. 75, pp. 71-80, 1996.

[8] E. Ha and W. Kim, "A study of low-power density laser welding process with evolution of free surface," Int. J. Heat Fluid Flow, vol. 26, pp. 613-621, 2005.

[9] Z. S. Saldi, A. Kidess, S. Kenjereš, C. Zhao, I. M. Richardson, and C. R. Kleijn, "Effect of enhanced heat and mass transport and flow reversal during cool down on weld pool shapes in laser spot welding of steel," Int. J. Heat Mass Trans., vol. 66, pp. 879-888, 2013.

[10] M. Akbari, S. Saedodin, D. Toghraie, R. Shoja-Razavi, and F. Kowsari, "Experimental and numerical investigation of temperature distribution and melt pool geometry during pulsed laser welding of Ti6A14V alloy," Opt. Laser Technol., vol. 59, pp. 52-59, 2014.

[11] K. Mundra , T. DebRoy, and K. M. Kelkar, "Numerical prediction of fluid flow and heat transfer in welding with a moving heat source", Numer. Heat Trans. A-Apl., vol. 29, pp. 115-122, 1996.

[12] A. Kidess, S. Kenjereš, and C. R. Kleijn, "The influence of surfactants on thermocapillary flow instabilities in low Prandtl melting pools," Phys. Fluids, vol. 28, pp. 062106, 2016.

[13] A. Kidess, M. Tong, G. Duggan, D. J. Browne, S. Kenjereš, I. Richardson, and C. R. Kleijn, "An integrated model for the post-solidification shape and grain morphology of fusion welds," Int. J. Heat Mass Trans., vol. 85, pp. 667-678, 2015.

[14] W. Cho, S. Na, C. Thomy, and F. Vollertsen, "Numerical simulation of molten pool dynamics in high power disk laser welding," J. Mater. Process. Technol., vol. 212, pp. 262-275, 2012.

[15] Z. Gao, P. Jiang, G. Mi, L. Cao, and W. Liu, "Investigation on the weld bead profile transformation with the keyhole and molten pool dynamic behavior simulation in high power laser welding, "Int. J. Heat Mass Trans., vol. 116, pp. 1304-1313, 2018.

[16] C. Panwisawas, Y. Sovani, R. P. Turner, J. W. Brooks., H. C. Basoalto, I. Choquet, "Modelling of thermal fluid dynamics for fusion welding," J. Mat. Proc. Tech., vol. 252, pp. 176-182, 2018.

[17] F. Rosler and D. Bruggemann. "Shell-and-tube type latent heat thermal energy storage: numerical analysis and comparison with experiments," Heat Mass Trans., vol. 47, pp. 1027-1033, 2011.

[18] OpenFOAM, The open source CFD toolbox, OpenCFD Ltd, 2009. Available: http://www.openfoam.com

[19] J. Yang, S. Sun, M. Brandt, and W. Yan, "Experimental investigation and 3d finite element prediction of the heat affected zone during laser assisted machining of Ti6Al4V alloy," J. Mater. Process. Tech, vol. 210, pp. 2215-2222, 2010.

[20] M. Boivineau, C. Cagran, D. Doytier, V. Eyraud, M. H. Nadal, B. Wilthan, and G. Pottlacher, "Thermophysical properties of solid and liquid ti-6al-4v (ta6v) alloy," Int. J. Thermophys., vol. 27, pp. 507-529, 2006. 
[21] K. C. Mills, Recommended values of thermophysical properties for selected commercial alloys, Cambridge, Woodhead Publishing Limited, 2002.

[22] J. Svenungsson, I. Choquet, and A. F. H. Kaplan, "Laser welding process - a review of keyhole welding modelling," Phys. Proc., vol. 78, pp. 182-191, 2015.

[23] S. Pang, L. Chen, J. Zhou, Y. Yin, and T. Chen, "A three-dimensional sharp interface model for self-consistent keyhole and weld pool dynamics in deep penetration laser welding," J. Phys. D: Appl. Phys., vol. 44, pp. 025301, 2011. 